\title{
Strong expression of TGF-beta in human host tissues around subcutaneous Dirofilaria repens
}

\author{
Norbert W. Brattig • Paul Racz • Achim Hoerauf • \\ Dietrich W. Büttner
}

Received: 23 March 2010 /Accepted: 9 August 2010 /Published online: 1 September 2010

(C) The Author(s) 2010. This article is published with open access at Springerlink.com

\begin{abstract}
Dirofilaria repens and other Dirofilaria species are widely distributed parasitic nematodes of carnivores, which occasionally are transmitted to men, causing subcutaneous nodules. In humans, it usually occurs only as single male or female filariae without production of microfilariae. The non-productive living or dead Dirofilaria worms in subcutaneous biopsies from 15 human patients permitted us to study the role of the pleiotropic and immunoregulatory cytokine transforming growth factor beta (TGF-beta) independent from the influence of microfilariae. Antiserum against latent TGF-beta 1 was used for an immunohistological examination. In the infiltrates around female and male filariae, there occurred strongly TGF-beta-positive macrophages, mast cells, endothelial cells, fibrocytes, and giant cells adjacent to dead worms. In one nodule, secondary lymph follicles were observed with clearly TGF-beta-positive B cells in the mantle zone and weakly positive macrophages and B cells in the germinal centre. A network of CD35-positive follicular dendritic cells was observed in the germinal centre. All Dirofilaria contained Wolbachia endobacteria, which probably had attracted the numerous TGF-beta-negative neutrophils near to the worm. Wolbachia were phagocytosed by neutrophils adjacent to dead filariae. Macrophages and lymphocytes expressed the
\end{abstract}

\footnotetext{
N. W. Brattig $(\bowtie) \cdot$ P. Racz $\cdot$ D. W. Büttner

Bernhard Nocht Institute for Tropical Medicine,

Bernhard-Nocht-Str. 74,

20359 Hamburg, Germany

e-mail: nbrattig@bni-hamburg.de

A. Hoerauf

Institute for Medical Microbiology,

Immunology and Parasitology, University of Bonn,

Sigmund-Freud-Str. 25,

53105 Bonn, Germany
}

MHC class II molecule HLA-DR in small accumulations of immune cells in the outer zone of the infiltrate and the mantle zone and germinal centre of secondary lymph follicles. It is concluded that single non-productive Dirofilaria worms elicit a strong expression of TGF-beta. This result is in accordance with observations on Onchocerca volvulus from patients with the hyporeactive (generalised) form.

\section{Introduction}

Nematodes of the genus Dirofilaria are widely distributed parasites of carnivores including domestic dogs. Mosquitoes occasionally transmit Dirofilaria to humans (McCall et al 2008; Simón et al. 2009), where they at first migrate subcutaneously (Franz et al. 1982) and finally live in a pseudocyst. After some time, that may last several years, the worms die releasing antigens leading to an increased immune reaction, which may be a medical indication for the excision of the nodule. Usually, human patients only host one non-productive female or male Dirofilaria and, only rarely, male and female worms are transmitted, and microfilariae are produced. Dirofilaria repens from subcutaneous nodules of 14 human patients were reported to harbour all Wolbachia endobacteria (Grandi et al. 2008), as many filaria species do. Wolbachia attract neutrophils and contribute to the inflammatory reactions (Brattig et al. 2001). The diagnostic histopathology of subcutaneous human dirofilariasis is described in several textbook articles and reports (e.g. Marty and Neafi RC 2000; Ratnatunga and de Witesundera 1999).

Filariae produce an orthologue of human transforming growth factor beta (TGF-beta), (Gomez-Escobar et al. 2000) and they elicit its expression in different cells of their host (Brattig et al. 2009; Korten et al. 2010). The three 
isoforms of TGF-beta are members of a family of evolutionarily conserved pleiotropic cytokines playing a critical role during embryogenesis and homeostasis of adult organisms. Disturbance of their metabolism has been associated with various diseases including cancer, autoimmune and cardiovascular diseases (Byrne et al. 2008; Gourmans et al. 2009). In infections, they can contribute to the immunosuppression, facilitating survival of parasites and other microbes, e.g. by inhibition of macrophages (Brattig, 2004; Hoerauf et al. 2005). But, they also may play a role in pro-inflammatory reactions, in neoangiogenesis, wound healing, and tissue modelling ( $\mathrm{Li}$ et al. 2006; Massagué 1990, Taylor 2009). Different immune cells produce the inactive latent TGF- $\beta$, which is in complex with latency-associated protein (LAP) alone or with LAP and latent TGF- $\beta$-binding protein, and which is secreted upon antigen recognition by the immune cells (Taylor 2009).

Previously, we reported on the expression of TGF-beta in human cells elicited by living and dead Onchocerca volvulus filariae using immunohistology (Brattig et al. 2009, Korten et al. 2010). In the present study, we compared these findings with immune reactions of human patients with dirofilariasis with regard to the expression of TGF-beta elicited by single worms not producing microfilariae.

\section{Patients, materials and methods}

\section{Biopsies from human patients}

Subcutaneous nodules with Dirofilaria sp. from 28 human patients, which had been sent for a parasitological diagnosis to the Bernhard Nocht Institute between 1983 and 2008, were examined using immunohistology. The filariae were acquired in the following countries: twice in France, twice in Spain, once in Tunisia, twice in Egypt, once in Turkey, twice in Ukraine, once in Russia, once in Kazakhstan, twice in India, four times in Sri Lanka, and ten times in the country not known. From 15 patients, enough sections were available for staining with antiserum against TGF-beta. The nodules had been fixed in formaldehyde solution, embedded in paraffin, and sections were stained with haematoxylin and eosin and often Giemsa. The diagnosis of the worms was based on the maximum width, the thick cuticle bulging inward in the region of the prominent lateral cords and the large body wall muscles. All observed Dirofilaria worms presented the characteristic external longitudinal cuticular ridges and hence, belonged to the subgenus Dirofilaria, excluding the subgenus Nochtiella. No Dirofilaria immitis was observed. Based on their morphology and the epidemiology, the worms were probably $D$. repens, but it cannot be excluded that one ore more of them belonged to another Dirofilaria species. Sex, viability and productivity of the worms were deter-
Fig. 1 Immunohistology of subcutaneous nodules with a single living nulliparous female (f) Dirofilaria sp. from human patients. A vital motile female worm in a pseudocyst (arrow; ep epidermis). Giemsa. Scale bar $=500 \mu \mathrm{m}$

Fig. 2 Immunohistology of subcutaneous nodules with a single living nulliparous female $(f)$ Dirofilaria sp. from human patients. Nodule with two secondary lymph follicles $(f o l)$ and defensin-positive neutrophils (arrowhead, inset) near to the pseudocyst (cyst), which contains the worm. Scale bar $=500 \mu \mathrm{m} ; 25 \mu \mathrm{m}$ (inset)

Fig. 3 Immunohistology of subcutaneous nodules with a single living nulliparous female $(f)$ Dirofilaria sp. from human patients. Neutrophils (arrowheads) adjacent to the cuticle $(\mathrm{cu})$ of a female worm with many DiWSP-positive Wolbachia endobacteria $(b a)$ in the hypodermis. Scale bar $=50 \mu \mathrm{m}$

Fig. 4 Immunohistology of subcutaneous nodules with a single living nulliparous female $(f)$ Dirofilaria sp. from human patients. Mature CD68-positive macrophages (long arrow) and neutrophils (arrowhead) near to the cuticle of a worm. A macrophage phagocytoses a neutrophil (short arrow). Scale bar $=50 \mu \mathrm{m}$

Fig. 5 Immunohistology of subcutaneous nodules with a single living nulliparous female $(f)$ Dirofilaria sp. from human patients. The pseudocyst containing the filaria is limited by a wall of neutrophils and TGF-beta-positive macrophages (long arrow) and in some distance positive vessels (short arrow). Scale bar $=50 \mu \mathrm{m}$

Fig. 6 Immunohistology of subcutaneous nodules with a single living nulliparous female $(f)$ Dirofilaria sp. from human patients. Detail of (5) showing TGF-beta-positive macrophages (arrow) and three positive blood vessels $(b v)$. Scale bar $=50 \mu \mathrm{m}$

Fig. 7 Immunohistology of subcutaneous nodules with a single living nulliparous female ( $f$ ) Dirofilaria sp. from human patients. TGF-betapositive macrophages near to the cuticle of a filaria from another patient. Scale bar $=50 \mu \mathrm{m}$

Fig. 8 Immunohistology of subcutaneous nodules with a single living nulliparous female $(f)$ Dirofilaria sp. from human patients. TGF-betapositive macrophages (long arrow) and negative neutrophils (arrowhead) near to the pseudocyst with the worm. Plasma cells (short arrows) are labelled by the anti-TGF-beta serum. Scale bar $=50 \mu \mathrm{m}$

Fig. 9 Immunohistology of subcutaneous nodules with a single living nulliparous female $(f)$ Dirofilaria sp. from human patients. Strongly TGF-beta-positive lymphocytes (short arrow), macrophages (long arrows) and endothelia of a blood vessel $(b v)$ in an accumulation of immune cells in the outer ring of the infiltrate. Scale bar $=50 \mu \mathrm{m}$

Fig. 10 Immunohistology of subcutaneous nodules with a single living nulliparous female ( $f$ ) Dirofilaria sp. from human patients. Clearly HLA-DR-positive macrophages (arrows) and plasma cells (arrowhead) in an accumulation of immune cells in the outer ring of the infiltrate. Scale bar $=50 \mu \mathrm{m}$

Fig. 11 Immunohistology of subcutaneous nodules with a single living nulliparous female (f) Dirofilaria sp. from human patients. Strongly TGF-beta-positive lymphocytes in the mantle zone (arrow) and weakly positive cells in the germinal centre (arrowhead) of one of the secondary lymph follicles shown in (Fig. 2). Scale bar $=50 \mu \mathrm{m}$

Fig. 12 Immunohistology of subcutaneous nodules with a single living nulliparous female $(f)$ Dirofilaria sp. from human patients. Many strongly CD35-positive follicular dendritic cells characteristic for secondary lymph follicles are found in the germinal centre (arrowhead) but not in the mantle zone (arrow). Scale bar $=50 \mu \mathrm{m}$

mined morphologically in the histological sections as described by Büttner et al. (1988) for O. volvulus. To assess the vitality, staining with antiserum against glutathione $\mathrm{S}$ transferase1 ( $O v$ GST 1) was also used (Hoerauf et al. 2003). The TGF-beta-stained 15 nodules contained nine living female filariae, three obviously dead females, one moribund 

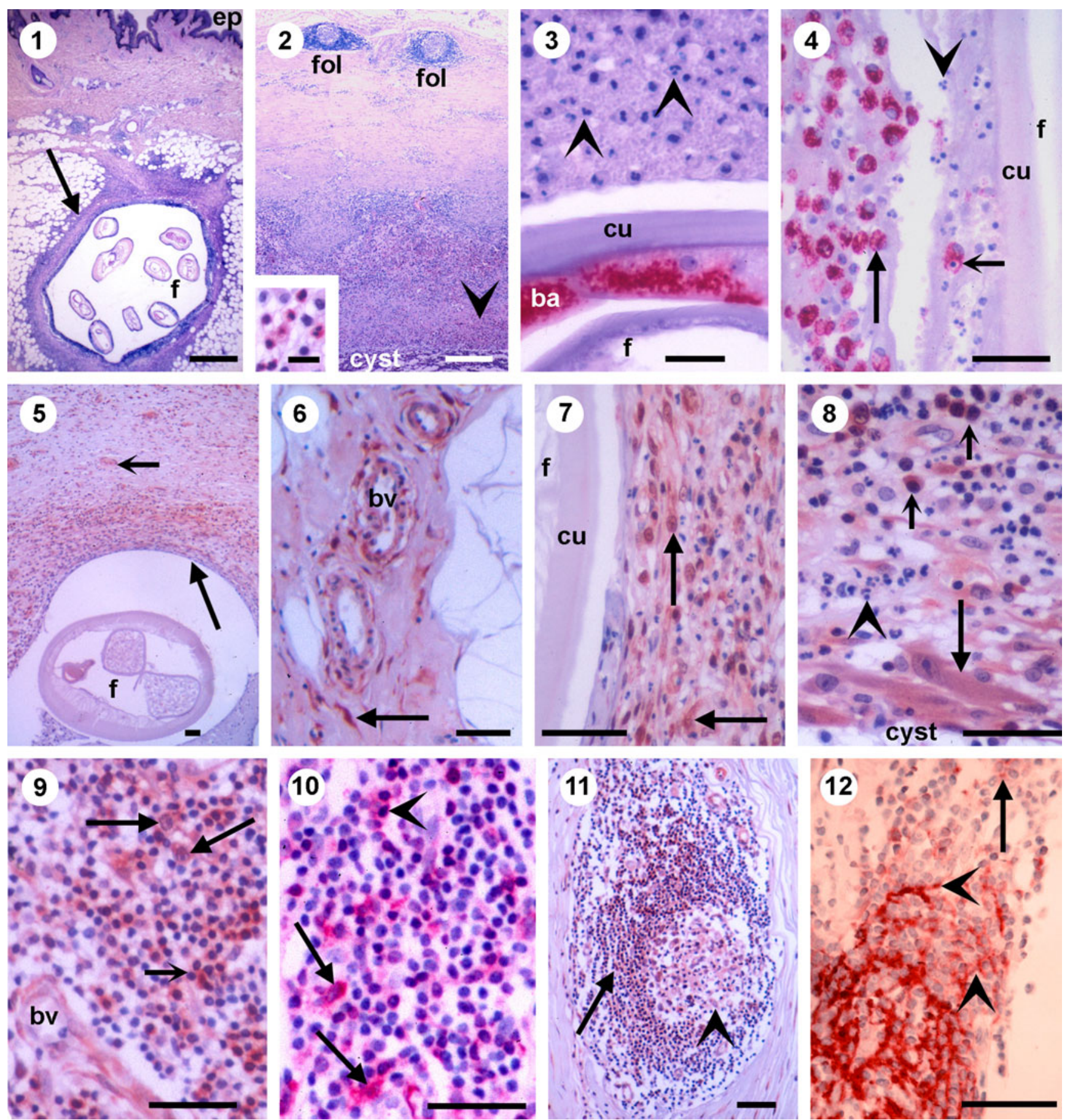

female (as defined by Duke et al. 2002) and two dead male worms. We assume that the patients had not been treated with any drug affecting Dirofilaria.

\section{Immunohistochemistry}

Dewaxed sections underwent antigen retrieval according to the advices of the manufacturers providing the primary antibody. For TGF-beta staining, the sections were incubated with polyclonal antibodies (pabs) produced in goats immunised with $\mathrm{CHO}$ cell-derived, recombinant human TGF-beta 1 latency-associated peptide (R\&D Systems, Wiesbaden, Germany, 1:200). A pre-diluted biotinylated anti-goat mouse monoclonal antibody (mab) served as a secondary antibody, and for visualisation, incubation with streptavidin-horseradish peroxides (HRP) and development with the brown chromogenic were used (HRP-AEC Cell \& Tissue Staining Kit, R\&D Systems). Later, when improved anti-human LAP 
antibodies became available, onchocercoma sections were stained to confirm the specificity of the goat pab. Nonspecific goat serum was applied for negative controls.

Monoclonal antibodies were used to characterise the immune cells: CD68 for mature macrophages (clone PGM1, 1:50), anti-L1 protein for immature macrophages (calprotectin, clone MAC387, 1:50), mast cell tryptase (clone AA1, 1:200), CD20 for B cells (clone L26, 1:50; all previous antibodies from DakoCytomation, Hamburg, Germany), major histocompatibility complex (MHC) class II molecule human leukocyte antigens (HLA)-DR (clone TAL.1B5, 1:30, R\&D Systems), defensin for neutrophils (1:4,000, Dianova, Hamburg, Germany), eosinophilic cationic protein for eosinophils (ECP EG2, 1:50, Kabi Pharmacia, NJ, USA), CD57 for NK cells (clone HNK-1, 1:20, Becton Dickinson, USA), CD3 for T cells (clone EP449E, 1:400, Epitomics, USA), and CD35 for follicular dendritic cells (clone RLB25, 1:60, Novocastra, Newcastle upon Tyne, UK). We applied pabs from rabbits against $D$. immitis Wolbachia surface protein to demonstrate Wolbachia endobacteria ( $D i$ WSP, 1:1,000, from Prof. Claudio Bandi, Università degli Studi di Milano, Milano; (Bazzocchi et al. 2000)), OvGST1 to determine the vitality $(1: 1,000$, from Prof. Eva Liebau, Department of Molecular Physiology, University of Münster) and IgE (1:2,000, DakoCytomation). For several mabs and the pabs, the alkaline phosphatase antialkaline phosphatase technique was applied according to the protocol from DakoCytomation. For CD3, CD20 and CD57 staining, the sections were pre-treated by boiling in citrate solution at $\mathrm{pH} 6$ and for CD35, in EDTA solution at $\mathrm{pH} 8$. For CD3 and CD35, AEC kits were used (PolyLink, Peroxydase Label HRP, DCS Innovative Diagnostik Systeme, Hamburg, Germany). Due to the limited availability of the nodules, not all sections from the nodules could be stained with the differentiation markers mentioned above.

\section{Results}

Host TGF-beta expression is elicited by living Dirofilaria

None of the living female worms contained embryos or microfilariae. The single vital and motile Dirofilaria worms lived in a pseudocyst (Fig. 1) containing some neutrophils or no cells. An inner cell wall of TGF-beta-negative and defensin-positive neutrophils (Figs. 2, 3, 4, and 5) adjoining an outer wall of strongly TGF-beta- and CD68-positive mature macrophages (Figs. 4, 5, 6, 7, 8) surrounded the pseudocyst. In the wall and in some distance, lymph and blood vessels with strongly TGF-beta-positive endothelial cells (Figs. 5, 6 and 9) and weakly positive vascular smooth muscle cells were found. The infiltrate also contained TGFbeta-positive fibrocytes and strongly TGF-beta-positive
Fig. 13 Immunohistology of subcutaneous nodules with dead or moribund female (f) Dirofilaria sp. from human patients. Granuloma with a dead immotile female filaria (arrow). HE. Scale bar $=500 \mu \mathrm{m}$ Fig. 14 Immunohistology of subcutaneous nodules with dead or moribund female $(f)$ Dirofilaria sp. from human patients. Another dead female worm with TGF-beta-positive macrophages (arrows) and negative neutrophils (arrowheads) near to the cuticle $(c u)$. Scale bar $=50 \mu \mathrm{m}$

Fig. 15 Immunohistology of subcutaneous nodules with dead or moribund female (f) Dirofilaria sp. from human patients. Another dead female worm with TGF-beta-positive macrophages (arrows) and negative neutrophils (arrowheads) near to the cuticle (cu). Scale bar $=50 \mu \mathrm{m}$

Fig. 16 Immunohistology of subcutaneous nodules with dead or moribund female $(f)$ Dirofilaria sp. from human patients. Another dead female worm with TGF-beta-positive macrophages (arrows) and negative neutrophils (arrowheads) near to the cuticle $(\mathrm{cu})$. In some distance of the filaria are TGF-beta-positive mast cells (arrows), which are IgE-positive (inset). Scale bar $=50 \mu \mathrm{m}$

Fig. 17 Immunohistology of subcutaneous nodules with dead or moribund female $(f)$ Dirofilaria sp. from human patients. Another dead female worm with TGF-beta-positive macrophages and negative neutrophils near to the cuticle. In some distance of the filaria are TGFbeta-positive mast cells which are IgE-positive, and lymph (short arrow) and blood vessels (long arrows) with strongly positive endothelia and weakly positive muscle cells. Scale bar $=50 \mu \mathrm{m}$

Fig. 18 Immunohistology of subcutaneous nodules with dead or moribund female $(f)$ Dirofilaria sp. from human patients. Another disintegrating female worm with TGF-beta-positive giant cells and macrophages (arrows) and negative neutrophils (arrowhead). Scale bar $=50 \mu \mathrm{m}$

Fig. 19 Immunohistology of subcutaneous nodules with dead or moribund female $(f)$ Dirofilaria sp. from human patients. Another disintegrating female worm with TGF-beta-positive giant cells and macrophages and negative neutrophils. Plasma cells (short arrow) are labelled by the anti-TGF-beta serum. Scale bar $=50 \mu \mathrm{m}$

Fig. 20 Immunohistology of subcutaneous nodules with dead or moribund female $(f)$ Dirofilaria sp. from human patients. A moribund or dead female filaria with morulae and degenerated microfilariae in the uterus is encircled by TGF-beta-positive giant cells (arrows) and negative neutrophils (arrowhead). Scale bar $=50 \mu \mathrm{m}$

Fig. 21 Immunohistology of subcutaneous nodules with dead or moribund female $(f)$ Dirofilaria sp. from human patients. A moribund or dead female filaria with morulae and degenerated microfilariae $(\mathrm{mf})$ in the uterus is encircled by TGF-beta-positive giant cells and negative neutrophils. Near to the female filaria with numerous Wolbachia endobacteria ( $b a, D i$ WSP-stained) in the hypodermis (hy) and in oocytes or zygotes in the uterus (arrow) are neutrophils with phagocytosed endobacteria and degenerating neutrophils are taken up by a giant cell. Scale bar $=10 \mu \mathrm{m}$

Fig. 22 Immunohistology of subcutaneous nodules with dead or moribund female (f) Dirofilaria sp. from human patients. A moribund or dead female filaria with morulae and degenerated microfilariae in the uterus is encircled by TGF-beta-positive giant cells and negative neutrophils. Near to the female filaria with numerous Wolbachia endobacteria ( $b a, D i$ WSP-stained) in the hypodermis $(h y)$ and in oocytes or zygotes in the uterus are neutrophils with phagocytosed endobacteria (arrows) and degenerating neutrophils are taken up by a giant cell (arrowheads). Scale bar $=10 \mu \mathrm{m}$

Fig. 23 Immunohistology of subcutaneous nodules with dead or moribund female $(f)$ Dirofilaria sp. from human patients. A moribund or dead female filaria with morulae and degenerated microfilariae in the uterus is encircled by TGF-beta-positive giant cells and negative neutrophils (arrowhead). Near to the female filaria with numerous Wolbachia endobacteria ( $b a, D i$ WSP-stained) in the hypodermis (hy) and in oocytes or zygotes in the uterus are neutrophils with phagocytosed endobacteria (arrows) and degenerating neutrophils are taken up by a giant cell. Scale bar $=10 \mu \mathrm{m}$ 

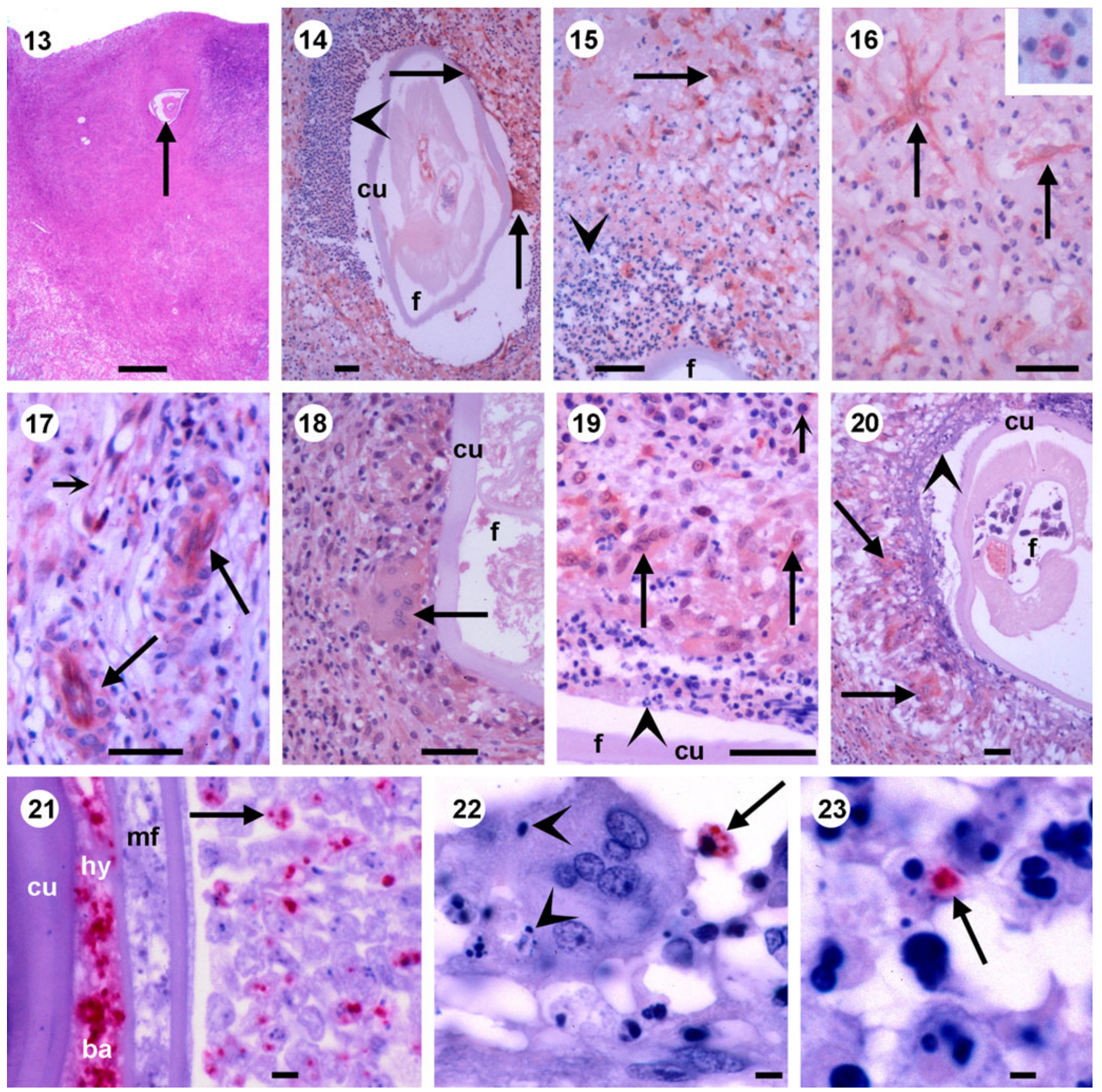

mast cells (Fig. 16) which had usually bound IgE to the cell surface as clearly seen in the inset (Fig. 16 inset). In the outer zone of the infiltrate were follicle-like accumulations of predominantly CD3-positive T cells and CD20-positive B cells interspersed between immature L1-protein- and CD68positive macrophages (not shown). Most of these lymphocytes were TGF-beta-negative, whereas the plasma cells were usually labelled by the TGF-beta serum (Figs. 8, 9), but they were not stained by the negative control serum. Dispersed macrophages and those in the cell accumulations were TGF-beta-positive (Figs. 6 and 9). Some of the macrophages and lymphocytes in the outer cell accumu- lations expressed the MHC class II molecule HLA-DR (Fig. 10). The frequency of eosinophils in the outer zone of the infiltrate varied much, and they were mostly scanty around vital worms in contrast to moribund and dead ones (not shown). All examined Dirofilaria from the 28 patients harboured Wolbachia endobacteria (Figs. 3 and 21).

Secondary lymph follicles

We observed in one biopsy with a living female Dirofilaria secondary lymph follicles presenting a mantle zone composed of $\mathrm{B}$ and some $\mathrm{T}$ lymphocytes and a germinal centre 
with B cells and some macrophages (Figs. 2, 11 and 12) indicating a strong local immune reaction of the host. A network of CD35-positive follicular dendritic cells, characteristic for secondary lymph follicles, was observed in the germinal centre (Fig. 12). Neutrophils were rare, and eosinophils were absent in the follicles. Many B cells in the mantle zone were moderately or strongly TGF-betapositive, whereas they were less positive in the germinal centre (Fig. 11). Macrophages and lymphocytes in the secondary lymph follicle expressed HLA-DR (not shown).

\section{Strong TGF-beta expression in response to dead filariae}

The host reaction around moribund and dead immotile Dirofilaria was a granuloma (Fig. 13) and not a pseudocyst containing the entire worm. Between the filaria and the wall of host cells was only a small artificial space (Figs. 14 and 20) or no space at all (Figs. 15 and 18). The wall of TGFbeta-negative neutrophils and positive mature CD68-positive macrophages was thicker (Fig. 14) than the wall around living worms. Many macrophages had fused to giant cells (Figs. 14, 18, 19 and 20), which we did not observe adjacent to living Dirofilaria. As in infiltrates around living worms, we observed TGF-beta-positive mast cells, which were also positive for IgE (Fig. 16), lymph and blood vessels (Fig. 17), fibrocytes and TGF-beta-labelled plasma cells (Fig. 19). The vascular muscle cells were less TGF-beta-positive than the endothelial cells (Fig. 17). TGF-beta expression in the immune cells was less around a disintegrating filaria. The frequency of eosinophils appeared to be higher in the infiltrates around the dead worms. One of the dead worms contained morulae and degenerated microfilariae in the uterus (Figs. 20, 21) that were no longer released.

Based on our experience with the morphology of $O$. volvulus, we assume that the observed dead Dirofilaria probably only had died shortly before their extirpation, and they still contained many morphologically intact Wolbachia endobacteria (Fig. 21). From these moribund or dead worms, Wolbachia were released and phagocytosed by neutrophils near the filaria (Figs. 22, 23). Giant cells were taking up neutrophils (Fig. 22), and these giant cells may present Wolbachia antigens causing immune responses to the endobacteria.

\section{Male Dirofilaria}

Only two nodules with a dead male filaria could be stained with antibodies against TGF-beta, and one living and two dead males were stained with other markers. A wall of neutrophils and CD68-positive mature macrophages, also expressing TGF-beta, surrounded the vital male worm (Fig. 24). And, in the outer zone, different immune cells were observed as described for female worms. The infiltrate contained many eosinophils positive for eosinophilic cationic protein (Fig. 25). The cells attached to the dead male worms were degenerated and TGF-beta-negative, but in some distance, were the same cell types as those around dead female worms that were strongly TGF-beta-positive (Figs. 26, 27).
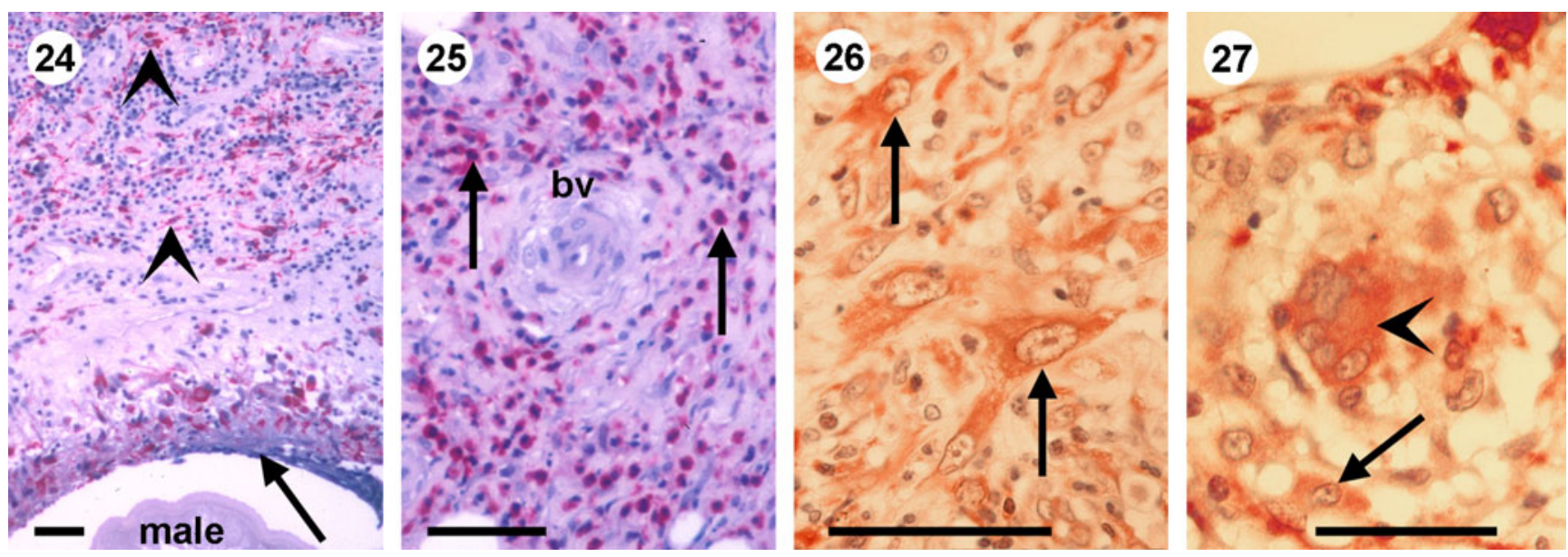

Fig. 24 Immunohistology of subcutaneous nodules with living or dead male Dirofilaria sp. from human patients. The wall around a living male Dirofilaria (arrow) contains neutrophils and mature CD68-positive macrophages, which also are seen in some distance of the worm (arrowhead). Scale bar $=50 \mu \mathrm{m}$

Fig. 25 Immunohistology of subcutaneous nodules with living or dead male Dirofilaria sp. from human patients. In the outer ring around the living male worm are numerous eosinophils (arrows) labelled by eosinophilic cationic protein. $b v$ blood vessel. Scale bar=50 $\mu \mathrm{m}$

Fig. 26 Immunohistology of subcutaneous nodules with living or dead male Dirofilaria sp. from human patients. The granuloma around a dead male Dirofilaria shows many strongly TGF-beta-positive macrophages (arrows) and some positive giant cells $($ arrowhead). Scale bar $=50 \mu \mathrm{m}$ Fig. 27 Immunohistology of subcutaneous nodules with living or dead male Dirofilaria sp. from human patients. The granuloma around a dead male Dirofilaria shows many strongly TGF-beta-positive macrophages (arrow) and some positive giant cells (arrowhead). Scale bar=50 $\mu \mathrm{m}$ 


\section{Discussion}

Vital nulliparous female Dirofilaria live in a pseudocyst, which is surrounded by a cell wall with neutrophils and macrophages. In contrast, live nulliparous female $O$. volvulus in onchocercomas are interspersed with the host tissues (Büttner et al. 1988; Specht et al. 2009), which contain numerous blood and lymph vessels. No or few neutrophils are attached to young $O$. volvulus. Only the motile anterior end of a female worm may lie in a small pseudocyst. Several older productive female $O$. volvulus may live in sometimes large cystic spaces without direct contact to the blood and lymph vessels. Often, plaques of degenerated neutrophils and macrophages are attached to portions of the cuticle adjoined by intact immune cells. In onchocercomas, neutrophils are attracted to the Wolbachia-containing worms (Brattig et al. 2001) and probably, this also applies to Dirofilaria. In contrast to dead worms, we did only find few eosinophils around living nulliparous Dirofilaria in accordance with the observations in onchocercomas with nulliparous filariae (Wildenburg et al. 1996). The cellular reactions to single dead female and male Dirofilaria resemble those observed around single dead O. volvulus. Already, Ratnatunga and de Witesundera (1999) observed secondary lymph follicles in Dirofilaria nodules. They show that a single non-productive Dirofilaria can elicit a strong local immune reaction as it is also indicated by the HLA-DR expression of antigen-presenting macrophages and B cells. In contrast, we found secondary lymph follicles only in onchocercomas with productive worms from hyperreactive patients (Brattig et al. 2010).

The expression of TGF-beta in the immune cells of the infiltrate around Dirofilaria corresponded to that observed in onchocercomas from hyporeactive immunosuppressed onchocerciasis patients (Brattig et al. 2009; Korten et al. 2010). The expression of TGF-beta adjacent to nonproductive female Dirofilaria is in accordance to its occurrence independently from embryogenesis and production of $O$. volvulus microfilariae. The functional role of TGF-beta as a mediator of immunosuppression is documented ( $\mathrm{Li}$ et al. 2006). TGF-beta promotes the differentiation of induced regulatory $\mathrm{T}$ cells (Treg) that has been demonstrated to be associated with the hyporeactive form of onchocerciasis (Korten et al. 2008). Activated Treg cells on their part secrete in an autocrine loop the latent form of TGF-beta 1 (Li et al. 2006). Thus, TGF-beta favours a hyporeactive status in the infected tissue. The described local hyporeactivity indicated by the TGF-beta expression exists in parallel with the strong inflammatory reaction indicated by the HLA-DR expression and the secondary lymph follicles in one nodule.

We assume that the TGF-beta expression in the immune cells is mainly elicited by the filariae. It does not depend on the Wolbachia since the depletion of the endobacteria by doxycycline treatment of onchocerciasis patients has no influence on TGF-beta (Korten et al. 2010). Notably, Dirofilaria as well as Onchocerca spp. and Wuchereria bancrofti generate themselves a TGF-beta 1 orthologue, but so far, we did not observe that the worm released this protein (Korten et al. 2009). Therefore, we assume that the filarial TGF-beta may have no influence on the host cells. However, we cannot exclude that factors involved in wound healing and angiogenesis may be involved (Folkman and Klagsbrun, 1987). As in onchocercomas (Korten et al. 2010), endothelial and muscle cells of blood vessels in the infiltrates around the Dirofilaria were clearly TGF-betapositive. The worms have probably elicited this or contributed to its expression. Thus, a recent report showed that $D$. immitis antigens could stimulate the synthesis of mediators in human endothelial cells (Simón et al. 2008; Simón et al. 2009). D. immitis antigens stimulate the generation of arachidonic acid metabolites including leukotriene B4 and prostaglandin E2, as well as the expression of the adhesion molecules ICAM-1 and PECAM-1. These mediators promote vasodilation and an activation of a permissive Th2 response. This response could furthermore contribute to diminish the fluids released from the vessels to the perivascular lung parenchyma. TGF-beta induced by endothelial cells as well as mediators of immune cells may promote the neovascularisation similar to what some tumours seem to do (Simón et al. 2008; Simón et al. 2009). TGF-beta plays a role for the maturation during angiogenesis (Armulik et al. 2010; Gourmans et al. 2009; Taylor, 2009). Further, we observed strongly TGF-betapositive endothelia in filaria-free tissues of prostate cancer (Büttner and Kaifi, unpublished). The expression of TGFbeta during maturation of new blood vessel elicited by vascular factors is well known from tumours (Felcht and Augustin, 2010) and other diseases (Gourmans et al. 2009).

In conclusion, single living female Dirofilaria not producing microfilariae, dead female and male worms elicit a strong TGF-beta pro-protein (LAP) expression in different immune cells of the human host.

Acknowledgments We thank Ingeborg Albrecht and Frank Geisinger for technical assistance. We are obliged to Prof. Claudio Bandi and Prof. Eva Liebau for the supply of antisera. We thank Dr. Klara Tenner-Racz (Bernhard Nocht Institute) for critically reading the manuscript.

Open Access This article is distributed under the terms of the Creative Commons Attribution Noncommercial License which permits any noncommercial use, distribution, and reproduction in any medium, provided the original author(s) and source are credited.

\section{References}

Armulik A, Abramsson A, Betsholtz C (2010) Endothelial/pericyte interaction. Circ Res 97:512-523 
Bazzocchi C, Jamnongluk W, O'Neill SL, Anderson TJ, Genchi C, Bandi C (2000) wsp gene sequences from the Wolbachia of filarial nematodes. Curr Microbiol 41:96-100

Brattig NW (2004) Pathogenesis and host responses in human onchocerciasis: impact of Onchocerca filariae and Wolbachia endobacteria. Microbes Infect 6:113-128

Brattig NW, Büttner DW, Hoerauf A (2001) Neutrophil accumulation around Onchocerca worms and chemotaxis of neutrophils are dependent on Wolbachia endobacteria. Microbes Infect 3:439-446

Brattig NW, Schwohl A, Hoerauf A, Büttner DW (2009) Identification of the lipid mediator prostaglandin $E_{2}$ in tissue immune cells of humans infected with the filaria Onchocerca volvulus. Acta Trop 112:231-235

Brattig N, Tenner-Racz K, Hoerauf A, Racz P, Korten S, Büttner DW (2010) Immunohistology of ectopic secondary lymph follicles in subcutaneous nodules from patients with hyperreactive onchocerciasis (sowda). Parasitol Res 107:657-666

Büttner DW, Albiez EJ, von Essen J, Erichsen J (1988) Histological examination of adult Onchocerca volvulus and comparison with the collagenase technique. Trop Med Parasitol 39:390-417

Byrne SN, Knox MC, Halliday GM (2008) TGF-beta is responsible for skin tumour infiltration by macrophages enabling the tumours to escape immune destruction. Immunol Cell Biol 86:92-97

Duke BOL, Marty AM, Peet DL, Pardon J, Pion SDS, Kamgno J, Boussinesq M (2002) Neoplastic change in Onchocerca volvulus and its relation to ivermectin treatment. Parasitology 125:431-444

Felcht M, Augustin HG (2010) Neue Erkenntnisse in der Tumorangiogenese. Klinikarzt 39:12-17

Folkman J, Klagsbrun M (1987) Angiogenic factors. Science 235:442-447

Franz M, Volkmer KJ, Lenze W (1982) A case of dirofilariasis in man (subgenus Nochtiella): a scanning electron microscope study. Tropenmed Parasitol 33:31-32

Gomez-Escobar N, Gregory WF, Maizels RM (2000) Identification of $t g h$ 2, a filarial nematode homolog of Caenorhabditis elegans daf-7 and human transforming growth factor beta, expressed in microfilarial and adult stages of Brugia malayi. Infect Immun 68:6402-6410

Gourmans M-J, Liu Z, ten Dijke P (2009) TGF-beta signalling in vascular biology and dysfunction. Cell Res 19:116-127

Grandi G, Morchon R, Kramer L, Kartenshev V, Simón F (2008) Wolbachia in Dirofilaria repens, an agent causing human subcutaneous dirofilariasis. J Parasitol 94:1421-1423

Hoerauf A, Mand S, Volkmann L, Büttner M, Marfo-Debrekyei Y, Taylor M, Adjei O, Büttner DW (2003) Doxycycline in the treatment of human onchocerciasis: kinetics of Wolbachia endobacteria reduction and of inhibition of embryogenesis in female Onchocerca worms. Microbes Infect 5:261-273

Hoerauf A, Satoguina J, Saeftel M, Specht S (2005) Immunomodulation by filarial nematode. Parasite Immunol 27:417-429

Korten S, Badusche M, Büttner DW, Hoerauf A, Brattig N, Fleischer B (2008) Natural death of adult Onchocerca volvulus and filaricidal effects of doxycycline induce local FOXP3+/CD4+ regulatory $\mathrm{T}$ cells and granzyme expression. Microbes Infect 10:313-324

Korten S, Büttner DW, Schmetz C, Hoerauf A, Mand S, Brattig N (2009) The nematode parasite Onchocerca volvulus generates the transforming growth factor-beta (TGF-beta). Parasitol Res 105:731-741

Korten S, Kaifi JT, Büttner DW, Hoerauf A (2010) Transforming growth factor beta expression by host cells is elicited locally by the filarial nematode Onchocerca volvulus in hyporeactive patients independently from Wolbachia. Microbes Infect 12:555-564

Li MO, Wan YY, Sanjabi S, Robertson AK, Flavell RA (2006) Transforming growth factor-beta regulation of immune responses. Annu Rev Immunol 24:99-146

McCall JW, Genchi C, Kramer LH, Guerrero J, Venco L (2008) Heartworm disease in animals and humans. Adv Parasitol 66:193-285

Marty AM, Neafi RC Dirofilariasis (2000) In: Meyers WM (ed) Pathology of infectious diseases. Vol. 1, Helminthiases, 2nd edn. Armed Forces Institute of Pathology, Washington D. C, pp 275-285

Massagué J (1990) The transforming growth factor-beta family. Annu Rev Cell Biol 6:597-641

Ratnatunga N, de Witesundera MS (1999) Histopathological diagnosis of subcutaneous Dirofilaria repens infection in humans. Southeast Asian J Trop Med Public Health 30:375-378

Simón F, Morchón R, Rodríguez-Barbero A, López-Belmonte J, Grandi G, Genchi C (2008) Dirofilaria immitis and Wolbachiaderived antigens: its effect on endothelial mammal cells. Vet Parasitol 158:223-231

Simón F, Morchón R, González-Miguel J, Marcos-Atxutegi C, SilesLucas M (2009) What is new about animal and human dirofilariosis? Trends Parasitol 25:404-409

Specht S, Brattig N, Büttner M, Büttner DW (2009) Criteria for the differentiation between young and old Onchocerca volvulus filariae. Parasitol Res 105:1531-1538

Taylor AW (2009) Review of the activation of TGF-\{beta $\}$ in immunity. J Leukoc Biol 85:29-33

Wildenburg G, Krömer M, Büttner DW (1996) Dependence of eosinophil granulocyte infiltration into nodules on the presence of microfilariae producing Onchocerca volvulus. Parasitol Res $82: 117-124$ 